\title{
Datenschutz und Medienbildung - Chancen und Barrieren in der schulischen Praxis
}

Andreas D. Schulz

\begin{abstract}
Die Digitalisierung im Bildungswesen und die zunehmende Relevanz von digitalen Bildungsmedien wirft verstärkt Fragen des Datenschutzes auf. Schülerinnen und Schüler, Eltern und Lehrkräfte waren mit dem Inkrafttreten der Europäischen Datenschutz-Grundverordnung 2019 verunsichert, welche Informationen erhoben, verarbeitet und gespeichert werden können. In den Klassenräumen entfernten die Lehrer die Geburtstagskalender und auf Schulfesten vermied man Fotos. Mit den Datenschutzerklärungen und den Verzeichnissen von Verarbeitungstätigkeiten betreten die Lehrkräfte Neuland. Die Digitalisierung der Bildung verändert nicht nur die Art des Unterrichts und die Kompetenzvermittlung, sondern auch die Strukturen und Prozesse innerhalb der Schule. Welchen Einfluss hat die Datenschutz-Grundverordnung in der Schule und wie beeinflusst sie die Digitalisierung der Lehr- und Lernprozesse? Wie wirken die rechtlichen Anforderungen, die sich entwickelnde Daten-Ökonomie bzw. Internetwirtschaft sowie die Mediatisierung der Schulgemeinschaft auf die Organisation des Unterrichts und der Schule?
\end{abstract}

\section{Einleitung}

Die Schließung der Schulen im Frühjahr 2020 verliehen digitalen Lehrund Lernformaten eine unvorhersehbare Aktualität und Bedeutung. Diese Entwicklung setzte sich mit dem digitalen Distanzunterricht im Winter 2021 fort und wird nachhaltig wirken. So wichtig der Start in das digitale Lernen auch war: Der Datenschutz spielte in dieser Zeit kaum eine Rolle (vgl. HKM 2020). Lehrerinnen und Lehrer erhielten in dieser Zeit kaum Hinweise, welche digitalen Lehr- und Lernangebote datenschutzkonform sind und wie die Datenschutzrechte der Schülerinnen und Schüler gewahrt werden können. Der Umgang der Schulen mit digitalen und datenschutzkonformen Lehr- und Lernangeboten variierte daher stark zwischen den Bundesländern. 
So wurden die Schulen während der Schulschließungen scheinbar in die Zeit des digitalen Lernens katapultiert. Die „Verwaltungsvereinbarung DigitalPakt Schule 2019 bis 2024“ unterstützt mit 5,5 Mrd. Euro die Schulen nahezu zum richtigen Zeitpunkt. Tatsächlich ist es bis zur Umsetzung des DigitalPaktes aber noch ein weiter Weg. In vielen Schulen sind weder Vereinbarungen über anstehende Maßnahmen (z.B. Zielvereinbarungen zur Nutzung der digitalen Ausstattung im Rahmen didaktischer Medienkonzepte) noch Gelder zur Finanzierung einer (ausreichenden) Breitbandanbindung an das Internet angekommen. Der DigitalPakt scheint den Schulen eine Option für eine "didaktische Wende“ mit Hilfe digitaler Lehr- und Lernformate zu bieten. Die Umsetzung wird allerdings noch einige Zeit benötigen. Dennoch werfen die Erfahrungen aus den Schulschließungen im Frühjahr 2020 und im Winter 2021 Fragen in Hinblick auf die Notwendigkeit einer aktuellen und für alle nutzbaren digitalen Infrastruktur, didaktischen Medienkonzepten, Medienkompetenzen, Medienbildung sowie den Datenschutz auf. Die Digitalisierung der Schulen beeinflusst direkt die Realisierung des Grundrechtschutzes auf informationelle Selbstbestimmung von LehrerInnen und SchülerInnen. Die Art und Weise der Nutzung digitaler Lehr- und Lernformate tangiert somit auch den Datenschutz. Das Chatformat ZOOM hat beispielsweise gezeigt, wie unsicher und anfällig digitale Anwendungen für Störungen von außen, wie z.B. die Teilnahme unbekannter Dritter, sind. Das Verhältnis von Medienbildung und Datenschutz in den Schulen ist mehr als prekär. Dieser Beitrag wird daher der Frage nachgehen, vor welchen Herausforderungen die Schulen im Rahmen der Realisierung einer datenschutzkonformen Medienbildung stehen. Welche Anforderungen seitens der Medienbildung und des Datenschutzes werden an die Schulen ${ }^{1}$ gestellt?

\section{Datenschutzrechtliche Relevanz und Anforderungen an die Medienbildung}

Welche Relevanz hat der Datenschutz in Schulen und welche Anforderungen müssen Schulen erfüllen? Die Frage nach der Relevanz des Datenschutzes in Schulen müsste eigentlich obsolet sein, denn die Mitglieder einer Schulgemeinschaft - Lehrkräfte, Eltern und Lernende - sind Bürger und Bürgerinnen, und sie haben daher natürlich alle Grundrechte wie auch das Recht auf informationelle Selbstbestimmung. Dennoch ergeben

1 Schule umfasst in diesem Beitrag alle Schulformen in Deutschland: z.B. Grundschulen, Haupt- und Realschulen, Gymnasien, berufliche Schulen etc. 
sich aus der pädagogischen Tätigkeit, der Bildungsinstitution und dem Erziehungs- und Schutzauftrag der Schule bezüglich der Kinder und Jugendlichen besondere Anforderungen. Schaumburg (2015) nennt in ihrer Studie sechs Einflussgrößen der digitalen Medien auf den Unterricht und den Schulalltag, die den Datenschutz direkt tangieren:

Die Schule wird erstens zunehmend beeinflusst durch die individuellen medialen Erfahrungen und Praxen der Kinder und Jugendlichen. Die regelmäßig veröffentlichten KIM und JIM Studien (vgl. Südwest, M. F. 2018, 2019) zeigen den Medienwandel und Medienkonsum der nachwachsenden Generation. Mobile Geräte wie Smartphones und Tablets werden in der Nutzung immer wichtiger. Die Kommunikation mit Freunden über Messengerdienste, die Informationssuche, Video- und Musikdienste sowie Online-Spiele nehmen einen wichtigen Platz im Medienverhalten ein. Die Alltagswirklichkeit von Kindern und Jugendlichen ist demnach nicht nur medialer, sondern auch digitaler geworden.

Schulen und Bildungsverlage versuchen sich auf diese Entwicklung einzustellen und den Kindern und Jugendlichen digitale Lernmöglichkeiten bereitzustellen. Schon vor der Corona-Pandemie standen eine Reihe von digitalen Werkzeugen zur Diagnose des Lernstandes, Leseprogramme (z.B. Antolin), Lernprogramme und Lernapps zur Verfügung. Mit den Schulschließungen haben sich diese Werkzeuge und Lernoptionen relativ stark verbreitet. Bildungsverlage und Anbieter von Apps stellen einerseits mehr Materialien auch kostenlos online. Andererseits werden von den Lehrkräften, die für die Lernenden Online-Materialien suchen, auch mehr Materialien nachgefragt. Eine zunehmende Verbreitung finden auch Online-Tests zur Diagnose von Lernfortschritten, die ebenfalls personenbezogene Daten beinhalten. Daher ist die Entwicklung eines Medienbildungskonzeptes für die weitere Digitalisierung der Schulen ebenso wichtig wie ein angemessenes Datenschutzkonzept. Denn durch die Nutzung externer digitaler Angebote sind immer auch Dritte eingebunden, die personenbezogene Daten erheben. Die Lehrkräfte müssen sich daher überlegen, welche externen digitalen Angebote genutzt werden, ob diese datenschutzkonform hinsichtlich der DSGVO sind, wie viele Einverständniserklärungen sie den Erziehungsberechtigten zumuten wollen und wie sie diese organisatorisch bewältigen können.

Die Digitalisierung wirkt sich drittens auch auf die technische und organisatorische Entwicklung der Schule als Institution aus. Die Ausstattung mit mobilen Geräten, digitaler Präsentationstechnik und WLAN sind zurzeit die größten technischen Herausforderungen für die Schulträger. Damit verbunden sind erhebliche Anstrengungen hinsichtlich der institutionellen Strukturen und Abläufe, z.B. die Organisation der Zugänge in das 
Internet, zu Lernplattformen und Schulportalen, die Umstellung der Administration und die Verteilung der digitalen Ressourcen und Geräte. Gleichzeitig mit dieser digitalen Vernetzung wächst auch hier der Bedarf an Datenschutzkonzepten.

Das oben beschriebene Medienverhalten von Kindern und Jugendlichen hat spezifische Ursachen. Neben der Kommunikation, Information und Entspannung nutzen die Heranwachsenden digitale Anwendungen auch zur Entwicklung ihrer eigenen Persönlichkeit. Entwicklungsaufgaben werden durch Kinder und Jugendliche zunehmend im Internet bearbeitet, wie z.B. die eigene Geschlechterrolle zu finden, ein eigenes Wertesystem aufzubauen, intellektuelle und soziale Kompetenzen auszubilden und einen eigenen Lebensstil zu entwickeln (vgl. Hurrelmann/Rosewitz/ Wolf 1985). Der Erziehungsauftrag der Schule diffundiert in die digitale Welt hinein und wird um digitale Kompetenzen erweitert, nicht nur um Kindern und Jugendlichen vor den Risiken im Internet zu schützen, sondern um sie bei der Suche nach eigenen Werten und Normen, dem Erlernen sozialer Kompetenzen, der Entwicklung eines eigenen Lebensstils und letztlich einer eigenen Persönlichkeit zu unterstützen. Das eigene Verhalten im Internet zu überprüfen, sich vor Gefährdungen zu schützen, personenbezogene Daten sparsam im Internet zu verbreiten und in Grundzügen auch die Internet- und Datenökonomie zu verstehen, sind Anknüpfungspunkte für eine Datenschutzbildung, die Bestandteil der Medienbildung in den Schulen sein sollte.

Hiermit sind auch die Gefahren und Risiken angesprochen, die mit der Nutzung digitaler Medien einhergehen können. Kinder und Jugendliche müssen sich mit den kommerziellen Interessen der Internetunternehmen, mit Aggression und Gewalt, sexuellen Übergriffen, rassistischen und verzerrten Informationen und Ratschlägen im Internet auseinandersetzen. Spitzer (2018) stellt eine ganze Reihe von gesundheitlichen, psychischen und gesellschaftlichen Risiken für Kinder und Jugendliche vor, die mit der Nutzung von Smartphones und dem Internet einhergehen sollen. Auch wenn seine Darstellungen sehr einseitig und plakativ sind, Bildungsinstitutionen müssen sich zunehmend mit dem Nutzen und den Gefährdungspotenzialen digitaler Medien auseinandersetzen und hierfür Bildungsinhalte für Kinder und Jugendliche entwickeln.

Die geschilderte Verbreitung digitaler Medien in den Bildungsinstitutionen zeigt den Bedarf an Medienbildung, der mit höheren Anforderungen hinsichtlich des Datenschutzes und einer Datenschutzbildung korrespondiert. Neben diesen pädagogischen Herausforderungen müssen sich Schulleitungen und Lehrkräfte mit den bestehenden gesetzlichen Anforde- 
rungen der Datenschutz-Grundverordnung auseinandersetzen und diese in den Schulen umsetzen.

Die Mitglieder einer Schulgemeinschaft besitzen dabei zunächst die gleichen Datenschutzrechte wie alle anderen Bürgerinnen und Bürger. Schulen haben möglichst datensparsam zu arbeiten und Informationen nur zweckgebunden zu erheben und zu speichern. Eltern haben im Auftrag ihrer noch nicht volljährigen Kinder das Auskunftsrecht, das Recht auf Korrektur und Löschung der Daten und sie können sich an den Datenschutzbeauftragten wenden. Die Schulen ernennen hierfür einen Datenschutzbeauftragten und eine Stellvertretung.

Darüber hinaus gelten die Bestimmungen des Sozial- und Gesundheitsdatenschutzes, z.B. für die Erhebung und Archivierung der Ergebnisse der Einschulungsuntersuchung oder anderen gesundheitlichen und psychologischen Untersuchungen. Zusätzlich gelten landestypische Verordnungen über die Verarbeitung personenbezogener Daten in Schulen. Diese beinhalten Vorschriften zum Umgang mit personenbezogenen Daten auf den privaten Endgeräten der Lehrkräfte, Vorgaben zum Führen der Klassenbücher und Kurshefte, Regelungen zum Führen der Schülerakten und Einsichtnahme der Eltern, Aufbewahrungspflichten, Art der zu sammelnden Daten (Schülerdaten, Daten der Lehrkräfte, Daten zum Unterricht und zu Schulveranstaltungen), Bestimmungen für die Übermittlung von Daten z.B. zwischen Kitas und Schule, bei Schulwechsel, zum Gesundheits- oder Jugendamt, aber auch Vorgaben zur Organisation des Datenschutzes (vgl. Verordnung über die Verarbeitung personenbezogener Daten in Schulen und statistische Erhebungen an Schulen vom 4.2.2009 in Hessen $^{2}$ ).

\section{Medienbildung als Datenschutzbildung}

Der Bildungs- und Erziehungsauftrag der Schulen, die Strukturen und Prozesse innerhalb der Schulen und der Datenschutz sind eng verknüpft. Eltern erhalten durch die Schülerinnen und Schüler die Einwilligungserklärungen über die Erhebung und Nutzung von personenbezogenen Daten und sie sind zunehmend sensibilisiert, wenn es um Aushänge und um Foto- und Videoaufnahmen geht. Aber auch im Unterricht müssen Lehrkräfte auf der Basis der Fachcurricula auf die Entwicklung von Medienkompetenzen achten. Im Folgenden sind die Anforderungen für die Primarstufe und die Sekundarstufe in Hessen beispielhaft aufgeführt:

2 Online verfügbar unter: https:/www.rv.hessenrecht.hessen.de/bshe/document/hev r-SchulStatErhVHEpAnlage1 (Abfrage am: 8.10.2020). 
- „Die Lernenden nutzen anforderungsbezogen unterschiedliche Medien gestalterisch und technisch. (...) Sie nutzen Neue Medien verantwortungsvoll und kritisch.“ (HKM 2011: 10)

- Die Lernenden sollen „einen selbstbestimmten Umgang mit sozialen Netzwerken im Spannungsfeld zwischen Wahrung der Privatsphäre und Teilhabe an einer globalisierten Öffentlichkeit praktizieren“ (HKM 2016: S. 9)

Die Grundlage für diese curricularen Anforderungen liefern Ansätze zur Medienbildung und Medienkompetenz, wie sie seit Baacke (1997) und Tulodziecki, Herzig und Grafe (2019) entwickelt wurden. In neueren Ansätzen wird vor allem auf eine höhere Medienbildung für Kinder und Jugendliche gesetzt. Medienbildung ist geeignet,

„sich diese (durch die digitalen Medien - A.S.) entfremdete Welt wieder anzueignen - und zu erkennen, dass sie von den Menschen selbst gemacht und verantwortet ist. Sie hat die Aufgabe, Bildungsprozesse zu unterstützen, die deutlich machen, wie Medien Werkzeuge der Menschen sind, um die Welt zu verstehen und über sie zu kommunizieren." (Moser 2019: 211)

Schülerinnen und Schüler sollen demnach wissen, welche Daten in welchem Kontext zu schützen sind und wie sie sich verhalten sollen, wenn Dritte auf diese Daten zugreifen und unbefugt verwenden. Neben den Grundzügen des Datenschutzes als Grundrechtsschutz sollen Schülerinnen und Schüler neben den allgemeinen Grundlagen (Passwortschutz, Schutzeinstellungen in Anwendungen und im Internet) auch lernen, was personenbezogene Daten sind, Verhaltensweisen zum Schutz ihrer personenbezogenen Daten kennen und sich vor Dritten schützen. Moser (2019) bewertet diese Ansprüche jedoch als Überforderung von Kindern und Jugendlichen:

„...) im schlimmsten Fall wird sogar die Illusion vermittelt, man habe es selbst in der Hand zu vermeiden, dass die eigenen Daten abgegriffen werden“" (Moser 2019, S. 213).

Daher sollte Medienbildung auch die Bedeutung systemrelevanter Akteure aufzeigen, wie z.B. die Rolle von Internetunternehmen, die Interessen und Möglichkeiten der Zivilgesellschaft sowie die Chancen durch staatliche und betriebliche Datenschutzbeauftragte. 


\section{Wirkungen der Triade aus Staat, Markt und Schulgemeinschaft}

Bislang wurden neben dem Medienverhalten von Kindern und Jugendlichen vorwiegend staatliche und medienpädagogische Perspektiven rezipiert und in Hinblick auf deren Wirkung für die Medienbildung und den Datenschutz bewertet. Im Folgenden soll die in der Literatur vorherrschende Perspektive erweitert werden. Eine am Datenschutz orientierte Medienbildung zeichnet sich durch die Berücksichtigung staatlichen Rechts und pädagogischer Leitlinien, durch das Antizipieren datenökonomischer und wirtschaftlicher Einflüsse auf das Handeln der Schulgemeinschaft und schließlich durch das soziale Handeln der Kinder, Jugendlichen, Eltern und Lehrkräften als Schulgemeinschaft aus. Die Umsetzung des Rechts auf informationelle Selbstbestimmung zeigt sich - nicht nur in der Schule - als Handlungsfolge aus einer Triade zwischen Hierarchie, Markt und informellem Handeln. Diese Hybridität (vgl. Evers/Rauch/Stitz 2002) kann einerseits positive Synergien für den Datenschutz ergeben, andererseits aber auch zu Spannungen (vgl. Schulz 2010) führen.

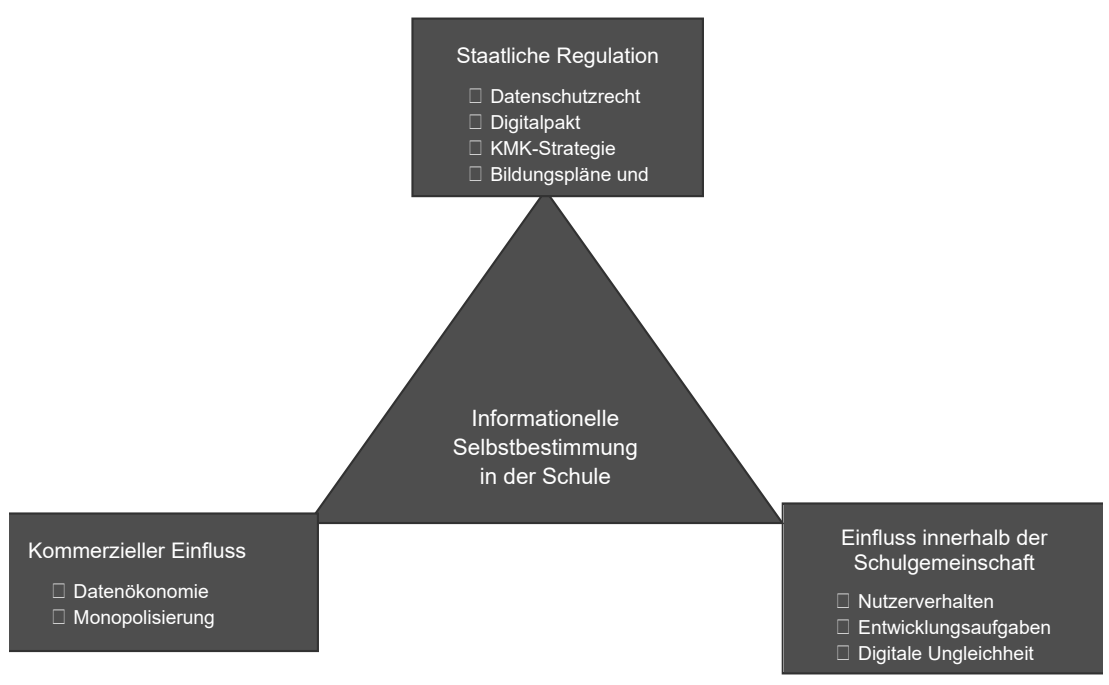

\section{Dezentralisierung und Flexibilität seitens des Staates}

Neben der DSGVO bestehen einige, schon zitierte Vorgaben der Kultusministerkonferenz und der Kultusministerien für den Datenschutz an den Schulen (z.B. Strategiepapier der KMK „Bildung in der digitalen Welt“, Kerncurricula, Digitalpakt). Deren Realisierung erfolgt allerdings weitge- 
hend dezentral. Das bedeutet, dass die Planung der technischen Ausstattung und die Medienbildungskonzepte in den Händen der Schulen liegen und die Umsetzung weitgehend selbstständig von den schulischen IT-Beauftragten mit Unterstützung der Schulämter und den städtischen Schulträgern getragen werden. Aufgrund der noch mangelhaften IT-Ausstattung konzentrieren sich die Bemühungen der Schulen vor allem auf eine Breitbandanbindung, WLAN-Ausstattung, Anschaffung von Präsentationshardware und eine Grundausstattung mit modernen Personalcomputern oder iPads. Ob für die Anschaffung mobiler Endgeräte im Sinne einer modernen Medienbildung noch genügend Ressourcen zur Verfügung stehen, ist fraglich. Hier wäre eine Balance zwischen der Entwicklung von Hardwarekonzepten und Medienbildungskonzepten sinnvoller. Auch die Integration der digitalen Medien in den Fachunterricht bleibt den einzelnen Schulen überlassen. Bestehende Apps und Angebote für Mathematik, Deutsch, Sprachen, Natur- und Gesellschaftswissenschaften müssen mit den fachlichen Kompetenzen verzahnt werden. Eine parallel stattfindende IT-Bildung kann Grundlagen für die Herausbildung von Medienkompetenzen bieten, aber führt kaum zu einer Selbstreflexion des eigenen Medienhandelns. Hinzu kommt eine Medienbildung, die den Datenschutz immer noch als individuelles Abwehrrecht vermittelt, andere Einflüsse wie traditionelle pädagogische Standards, die Datenökonomie und soziale Faktoren aber außen vorlässt. Der morgendliche Erzählkreis in den Grundschulen, Plakate mit Bildern vom Urlaub, Geburtstagskalender, Aushänge von Klassenfotos, Aufzeichnung von Schulfesten und Abbildungen errungener Siege bei Meisterschaften in der Schülerzeitung werden hinsichtlich des Datenschutzes zunehmend hinterfragt und bergen für die Verantwortlichen Unsicherheiten. Einfache Leitlinien für die in den meisten Fällen rechtlichen Laien, einheitliche Verzeichnisse der Verarbeitungstätigkeiten und eine reduzierte Form von Einverständniserklärungen für die zunehmende Anzahl von digitalen Anwendungen in der Schule fehlen aufgrund einer zunehmenden Autonomie und Flexibilität für die einzelnen Schulen.

\section{Der Datenmarkt und Einflüsse digitaler Monopole}

Der Markt wirkt mehrfach auf das Handeln der Schulen und die Umsetzung der Medienbildung. Zum einen tragen die Schülerinnen und Schüler regelmäßig neue technische Entwicklungen und Anwendungen in die Schule. Abgesehen von den Tablets und Smartphones treten bestimmte Anwendungen als Moden auf, mit denen sich die Schule auseinandersetzen muss. WhatsApp, Instagram, Tik Tok, Pokemon oder Fortnite. begeistern viele Kinder und Jugendliche, bergen aber auch Gefahren. Die Aufga- 
be der Schulen ist es daher, den Schülerinnen und Schülern zu vermitteln, dass diese Angebote mit den persönlichen Daten bezahlt werden und das problematisch werden kann. Wikipedia ist neben YouTube und Google eine der beliebtesten Wissensquellen. Doch die Zuverlässigkeit der Informationsangebote ist in vielerlei Hinsicht sehr unterschiedlich und sollte den Schülerinnen und Schülern bekannt sein. Hinzu kommen altersspezifische Unterschiede bei der Nutzung des Internets. Für Grundschüler und -schülerinnen existieren als Alternative zu Google und Wikipedia eine Vielzahl an altersgerechten Suchseiten und Kinderlexika im Internet (z.B. helles-köpfchen, frag-finn). Auf diese Informationsangebote muss besonders hingewiesen werden. Eine größere Herausforderung stellen digitale Anwendungen dar, die erst noch eine höhere Verbreitung finden werden. Die Smart Watch oder die Fitness Tracker, Smart Home, smarte Kleidung oder das Smart Car sind Beispiele für das Internet der Dinge, die besondere Anforderungen an den Datenschutz und die Medienbildung stellen (vgl. Husemann/Pittroff/Schulz 2018). Eine empirische Erhebung an der Universität Kassel 2017 zeigte, dass Schülerinnen und Schülern zu diesem Zeitpunkt kaum bewusst war, dass der Schlafzyklus, der Stromverbrauch, die Anzahl der Schritte am Tag, die gefahrenen Kilometer o.ä. ebenfalls personenbezogene Daten sein können und damit schützenswert sind. ${ }^{3}$ Digitale Anwendungen und Geräte können durch das Aufzeichnen, das Verarbeiten und die kommerzielle Nutzung personenbezogener Daten unser Verhalten im Alltag beeinflussen. Die Aufgabe der Schule wird daher auch hier darin bestehen, über die Funktionsweise des Internet der Dinge aufzuklären.

Hinzu kommen Markteinflüsse auf die Schule selbst. In den meisten Fällen legen die Schulträger fest, welche digitalen Medien Einzug in die Schulen finden. Doch häufig sind Betriebssystem und Anwendungen vorbestimmt - und das ist meist das Betriebssystem von Microsoft. Bei den Office-Lösungen gibt es eine breitere Varianz, allerdings scheint sich auch hier der Trend zu Microsoft durchzusetzen. In Hessen wurde der Einsatz von Office 365 zwar zunächst gestoppt. Der Anreiz, das Produkt vom Marktführer kostenlos einzusetzen, ist aber groß. Ähnliche Tendenzen bestehen bei bestimmten Lernprogrammen, die sich - sicherlich auch aus gutem Grund - in vielen Schulen durchzusetzen scheinen. Antolin, Anton,

3 Die Daten wurden erhoben im Rahmen des von Jörn Lamla und Alexander Roßnagel geleiteten interdisziplinären Projektes „Smart Environment, Smart Information?" (SEnSI) an der Universität Kassel (gefördert vom BMJV 01/2017 bis $12 / 2017)$. 
die Lernwerkstatt oder PADLET sind nur einige Beispiele. Auch die Schulbuchverlage setzen verstärkt auf Lernapps. Während der Schulschließungen im Corona Lockdown stellten die Verlage Megabyte an PDFs zur Verfügung, die Lernapps blieben aber teilweise kostenpflichtig. Kostenlose Versionen hatten nur eine eingeschränkte Funktionalität oder einen begrenzten Umfang. Der Datenschutz spielte zu Beginn der Pandemie kaum eine Rolle. Die Prämisse lag auf der Ermöglichung des Lernens zu Hause. Wie häufig personenbezogene Daten ungeschützt und ohne Aufklärung versandt und verarbeitet wurden, ist allerdings unklar. Digitales Lernen benötigt einen Zugang zu datenschutzkonformen Lernmaterialien. Den Schulen bleibt es dagegen selbst überlassen, welche digitalen Medien und Anwendungen sie erwerben und wie sie den Datenschutz gewährleisten. Das ist allerdings häufig jedoch eine Frage der Kompetenzen der schulischen Datenschutzbeauftragten, IT-Beauftragten oder interessierten Lehrerinnen und Lehrern.

\section{Die Schulgemeinschaft}

Aufgrund der hohen formalen Einbindung der Schule in die Hierarchie aus Kultusministerium und Schulämtern bzw. Bezirksstellen besteht die Gefahr, die Schule auch als demokratische und gemeinschaftliche Institution zu unterschätzen. Doch den Eltern steht eine Reihe von Mitsprachemöglichkeiten z.B. über die Elternbeiräte oder Fördervereine zu. Kinder lernen als Schulsprecher, in Klassenräten und Kinderkonferenzen demokratische Grundlagen kennen. Die Eltern gestalten zusammen mit den Lehrerinnen und Lehrern in Schulkonferenzen wesentliche Entwicklungen mit. In den Gesamtkonferenzen, Klassenkonferenzen und durch die Personalvertretung haben auch die Lehrerinnen und Lehrer einen gewissen Einfluss auf die Schul- und Unterrichtsorganisation.

Wesentliche strukturelle Veränderungen des Lehrens und Lernens sind in diesen Institutionen der Schulgemeinschaft zu besprechen und demokratisch zu legitimieren. Das gilt auch für den Datenschutz und die Medienbildung. Die Anschaffung digitaler Geräte und die Medienbildungskonzepte müssen in den demokratischen Gremien besprochen und abgestimmt werden. Die Datenschutzerklärungen und die Bedingungen für die Nutzung der Privatgeräte für dienstliche Zwecke (Passwortschutz, Virenscanner, Datensicherheit) sind vorzustellen und zu unterschreiben. Allerdings treten schon hier Probleme auf. Den Lehrkräften stehen keine dienstlichen Endgeräte wie Laptops oder iPads und nur selten dienstliche E-Mails zur Verfügung, d.h. die Unterrichtsvorbereitungen und die Zeugniserstellung werden meist am heimischen, privaten PC erstellt, was daten- 
schutzrechtliche Fragen aufwirft. In Zeiten von Corona werden die privaten Geräte zudem für das Online-Lernen mit Lernplattformen und für Videokonferenzen und E-Mails genutzt. Eine Vermischung von privaten und dienstlichen Daten, ein unzureichender Datenschutz und Datensicherheit sowie begründete oder unbegründete Ängste in Bezug auf Schäden bei privaten Geräten führen zu fehlender Akzeptanz für die Nutzung digitaler Möglichkeiten für die digitale Lehre und letztlich die Ablehnung der Medienbildung. Die verbreitete Annahme, dass nur jüngere Lehrkräfte den Zugang zu den digitalen Medien finden und diese im Unterricht einsetzen, kann nicht bestätigt werden. Den „digital native“ gibt es nicht (vgl. z.B. Schaumburg 2015). Individuelle Präferenzen und Interessen, aber auch eine unzureichende Medienbildung während der Ausbildung führen auch bei Jüngeren zu einem angespannten Verhältnis zu digitalen Lehrund Lernmitteln.

Neben der Schul- und Unterrichtsorganisation bestehen Anforderungen für den Datenschutz in Bezug auf die soziale Interaktion zwischen den Kindern und Jugendlichen. Zum ersten ist es für die Einzelnen zunehmend schwerer, sich digitalen Moden zu entziehen. Das betrifft die Anschaffung neuer Geräte, für deren Kosten ältere Schülerinnen und Schüler vermehrt Nebentätigkeiten aufnehmen, aber auch welche Apps en vogue sind. Kostenlose Spiele werden schnell zu Kostenfallen, wenn die darin versteckten App-In-Käufe ein höheres Prestige und ein schnelleres Weiterkommen im Spiel ermöglichen. Können Kinder und Jugendliche zwischen Online-Spielen und Lernapps auswählen, werden die Spiele und das „Zocken" präferiert, so dass sich letztlich alle Kinder im Jump-and-Run Modus befinden. Angebote wie z.B. Antonin, Anton, die Lernwerkstatt oder das „Internet-ABC“, eine Plattform zum Erlernen grundlegender Kompetenzen am Computer und im Datenschutz, nutzen freiwillig nur wenige Kinder und Jugendliche. Diese Angebote können daher nur in unterrichtsadäquater Form vermittelt werden. Die Nutzung des Internets als Informationsquelle hängt zudem stark mit dem Bildungsniveau des Elternhauses zusammen (vgl. Schaumburg 2015). Zweitens führt die Ausbreitung der digitalen Geräte zunehmend zu Problemen wie Cyber-Mobbing, Sexting und anderen Gefahren (vgl. Südwest 2019). Während Ausgrenzungen in vordigitalen Zeiten auf dem Schulhof blieben, besteht nun die Gefahr, dass die Betroffenen auch durch Schulwechsel oder bis ins Erwachsenenalter von den Angriffen begleitet werden. Eine früh ansetzende Medienbildung zum Schutz der Grundrechte, aber auch geeignete Konzepte an den Schulen gegen Cyber-Mobbing, Sexting und andere Gefahren müssen Teil der Medienbildung werden. Ein aus fürsorglicher Perspektive verständliches Han- 
dyverbot an Schulen (vgl. Spitzer 2012, 2018) verhindert die Risiken und Gefahren nur symptomatisch.

\section{Fazit: Anforderungen an die schulische Medienbildung aus der Perspektive des Datenschutzes}

Abschließend lassen sich einige Anforderungen für das Bildungswesen formulieren, die den Datenschutz für Kinder und Jugendliche, Eltern, Fürsorgetragende und die Lehrkräfte verbessern könnten. Diese Liste ist aber weder vollständig noch garantiert sie einen allumfänglichen Datenschutz:

1. Die Schulen können das Grundrecht auf informationelle Selbstbestimmung nicht alleine mit ihren begrenzten Ressourcen garantieren. Sie unterliegen zu vielen staatlichen, ökonomischen und informellen Einflüssen. Die Lehrkräfte sind Experten für Pädagogik und Erziehung, für zusätzliche Aufgaben braucht es Ausbildung oder schulübergreifende Beauftragte.

2. Schulträger und Schulaufsichtsbehörden könnten den Schulen konkrete Handlungsleitlinien und Hinweise für gute digitale Lehr- und Lernanwendungen bieten, die datenschutzkonform und pädagogisch wertvoll sind.

3. Digitales Lernen kann Bildungsungleichheiten aufgrund unzureichender technischer Ausstattung, geringer digital literacy oder bestehender Bildungsungleichheiten zwischen den Elternhäusern verstärken. Hier braucht es Unterstützung zum Beispiel durch die Bereitstellung von mobilen Endgeräten und WLAN, Kurse für Kinder und Eltern, die Einbindung der digitalen Lernformen in den Unterricht und in die Ganztagsbetreuung.

4. Um die Bedeutung des Datenschutzes zu erhöhen, muss das Verständnis über den Datenschutz, Handlungsmöglichkeiten und kritische Entwicklungen im Mediensystem in die Medienbildung einfließen. Im Rahmen der Schulcurricula können digitale Lehr- und Lernformen in den Fachunterricht integriert werden.

5. Die Schulaufsichtsbehörden sollten Datenschutzbeauftragte für mehrere Schulen installieren, die angesprochen werden können. Zwar sind die bisherigen schulischen Datenschutzbeauftragten formal unabhängig, doch eine Lehrkraft kann gegenüber der Schulleitung nicht zugleich weisungsgebunden und unabhängig sein.

6. Das Bildungswesen benötigt eine "digitale Wende“, die die Bedeutung für den Datenschutz und die Medienbildung im Sinne digitaler Lern- 
und Lehrformen stärkt. Bislang stehen vor allem die digitale Schuladministration, Breitbandanbindung und Präsentationstechniken im Vordergrund. Datenschutz ist im Interesse der Schülerschaft und Elternschaft und damit der Schüler- und Elternbeiräte. Aber auch andere bürger- und zivilgesellschaftliche Interessensgemeinschaften, die sich für die demokratische Digitalisierung der Gesellschaft einsetzen, sind hier gefordert.

Digitale Lehr- und Lernmittel wie auch die Digitalisierung der Schulprozesse müssen datenschutzkonform entwickelt werden. Das bedeutet einerseits, Lehrkräfte und die Kinder und Jugendlichen mit Fragen des Datenschutzes vertraut zu machen und dafür zu sensibilisieren, dass die Erhebung, Verarbeitung und Nutzung personenbezogener Daten ihre Grundrechte tangiert. Andererseits muss auch ein pragmatischer Umgang mit den rechtlichen Anforderungen, des Einflusses der Datenökonomie und der Mediatisierung der Schulgemeinschaft gefunden werden, der Lehrkräfte, Eltern, Kinder und Jugendliche nicht überfordert, gleichzeitig aber den Datenschutz gewährleistet. Hierfür braucht es die Entwicklung von Bildungsangeboten zum Thema Datenschutz und Medienbildung. Die Zivilgesellschaft ist zunehmend gefordert, den Prozess der Digitalisierung im Bildungswesen zu begleiten.

\section{Literatur}

Baacke, Dieter (1997): Medienpädagogik. Tübingen: Niemeyer.

Evers, Adalbert / Rauch, Ulrike / Stitz, Uta (2002): Von öffentlichen Einrichtungen zu sozialen Unternehmen: Hybride Organisationsformen im Bereich der Dienstleistungen. Berlin: Ed. Sigma.

Hessisches Kultusministerium (HKM) (2011): Bildungsstandards und Inhaltsfelder Das neue Kerncurriculum für Hesse, Primarstufe: Deutsch.

Hessisches Kultusministerium (HKM) (2016): Kerncurriculum gymnasiale Oberstufe, Deutsch.

Hessisches Kultusministerium (HKM) (2020). Digitale Kommunikation von Lebrkräften öffentlicher Schulen in Hessen während der Zeit der Covid-19-Pandemie. Hinweise des Hessischen Beauftragten für Datenschutz und Informationsfreiheit vom 23. März 2020.

Hurrelmann, Klaus / Rosewitz, Bernd / Wolf, Hartmut K. (1985): Lebensphase Jugend: eine Einführung in die sozialwissenschaftliche Jugendforschung. Weinheim u.a.: Juventa-Verlag. 
Husemann, Charlotte / Pittroff, Fabian / Schulz, Andreas D. (2018): Fitness-Tracking als Informationsproblem. Zu den Potenzialen und Herausforderungen rechtlicher Regulierung und pädagogischer Vermittlung. In: Datenschutz und Datensicherheit Dud 11, S. 694-700.

Moser, Heinz (2019): Einführung in die Medienpädagogik: Aufwachsen im digitalen Zeitalter, Wiesbaden. Wiesbaden: Springer VS.

Schaumburg, Heike (2015): Chancen und Risiken digitaler Medien in der Schule. Medienpädagogische und-didaktische Perspektiven. In: Bertelsmann-Stiftung (Hg.): Individuell fördern mit digitalen Medien - Chancen, Risiken, Erfolgsfaktoren. Gütersloh: Bertelsmann-Stiftung, S. 20-94.

Schulz, Andreas D. (2010): Organisationen zwischen Markt, Staat und Zivilgesellschaft: Arbeitsmarktförderung von Langzeitarbeitslosen im Deutschen Caritasverband. Wiesbaden: VS Verlag für Sozialwissenschaften.

Schulz, Andreas D. (2019): Informationelle Selbstbestimmung. Grundrecht oder Mythos? In: Praxis Politik 5, S. 42-48.

Spitzer, Manfred (2012): Digitale Demenz: Wie wir uns und unsere Kinder um den Verstand bringen. München: Droemer.

Spitzer, Manfred (2018): Die Smartphone-Epidemie: Gefahren für Gesundheit, Bildung und Gesellschaft. Stuttgart: Klett-Cotta.

Südwest, Medienpädagogischer Forschungsverbund (2018): KIM-Studie: Kinder und Medien, Computer und Internet. Stuttgart: MPFS.

Südwest, Medienpädagogischer Forschungsverbund (2019): JIM-Studie: Jugend, Information, (Multi-)Media. Stuttgart: MPFS.

Tulodziecki, Gerhard / Herzig, Bardo / Grafe, Silke (2019): Medienbildung in Schule und Unterricht: Grundlagen und Beispiele. Bad Heilbrunn: Verlag Julius Klinkhardt.

Zillien, Nicole (2009): Digitale Ungleichbeit: Neue Technologien und alte Ungleichbeiten in der Informations- und Wissensgesellschaft. Wiesbaden: VS Verlag für Sozialwissenschaften. 